Ann. Biol. anim. Bioch. Biophys., 1979, 19 (3 B), 733-738.

\title{
Mesure du débit du sang de la veine porte au moyen d'un débitmètre électromagnétique chez la chèvre
}

\author{
par J. L. BARRY, Elisabeth DEBRAS, J. LEFAIVRE *, C. CHAMPREDON \\ Laboratoire d'Ełude du Métabolisme Azoté \\ * Laboratoire d'Etude de la Digestion des Ruminants \\ I. N. R. A., Theix, St-Genès-Champanelle, \\ 63110 Beaumont.
}

Summary. Measurement of portal vein blood flow in goats using an electromagnetic flowmeter.

When quantitatively measuring the products of digestion in the portal blood, the following factors are to be studied :

- the difference between the concentrations of those products in the arterial blood afferent to the alimentary canal and of those in the venous portal blood,

- blood flow in the portal vein.

As a preliminary step, we have undertaken the study of the continuous measurement of the portal blood flow in ruminants (goats), using a method analogous to that employed by Rérat in pigs. Nine goats were fitted with a ring-probe around the portal vein ; during reading, the probe was attached to an electromagnetic flow-meter connected to a recorder. Fifteen readings were taken on 4 of the animals (mean live weight : $47 \mathrm{~kg}$ ) for about 7 hours after the morning feeding. After installation, each gauge allowed readings for 4 to 5 consecutive weeks. The signal then faded, and the animals were slaughtered and examined. As the wall of the portal vein had slightly thickened and therefore its lumen was only slightly reduced, signal damping could be attributed to a change in probe functioning. The mean flow, measured at the time of reading, was $1830 \mathrm{ml} / \mathrm{min}$ or $39 \mathrm{ml} / \mathrm{min} / \mathrm{kg}$ of live weight. This study showed a large variability in the flow and distinguished 3 variations throughout the day:

- rapid variations reaching $\pm 45 \mathrm{p}, 100$ over a period of a few seconds,

- variations reaching \pm 40 p. 100 over a period of a few minutes,

- large variations throughout the day, the mean maximum measurement being susceptible to reach 150 p. 100 of that of the minimum measurement.

The mean amount of flow varied up to \pm 25 p. 100 in the same animal from one day to another, and differed among the animals.

A general phenomenon occurred after the meal : the flow usually decreased by 10 p. 100 after about $1 \mathrm{hr}$; it then increased to attain approximately 115 p. 100 of that of the preprandial values towards the end of the reading.

The variable character of portal blood flow necessitates continuous reading while the quantitative occurrence of nutriments in the portal blood is being measured. 
L'évaluation du bilan des phénomènes digestifs chez le ruminant nécessite la mesure quantitative de l'apparition des nutriments dans le sang veineux porte : celà suppose connus simultanément d'une part les différences de concentration entre le sang veineux porte drainant le tube digestif ef le sang artériel afférent ef d'autre part le débit de sang de la veine porte.

La difficulté majeure de ce type d'étude est la mesure du débit sanguin porte. La plupart des méthodes utilisées jusqu'alors font appel à l'emploi d'un traceur injecté de manière discontinue ou continue dans la veine porte. Ces méthodes, parfois difficiles à utiliser dans des conditions physiologiques, ne permettent la mesure du débit qu'un nombre restreint de fois dans la journée. L'emploi de la technique à effet Doppler (Carr et Jacobson, 1968) présente l'inconvénient majeur de nécessiter l'estimation post-mortem du diamètre intérieur du vaisseau. Le débitmètre électromagnétique permet, en revanche, d'enregistrer directement le débit sanguin, en continu, sans perturber l'animal. Il a été utilisé avec succès chez le porc (Rérat, 1971). Kedenburg et Hübner (1973) l'ont employé chez le mouton, mais n'ont publié que des résultats sommaires. Nous avons voulu vérifier si l'importance des variations du débit sanguin porte chez le ruminant justifie l'emploi de cette méthode de mesure.

\section{Matériel et méthodes.}

- Animaux et préparation chirurgicale. - 9 chèvres adultes de race alpine, pesant de 40 à $60 \mathrm{~kg}$, sont utilisées. Après laparotomie sous anesthésie générale, un capteur électromagnétique d'un diamètre interne de $14 \mathrm{~mm}$, préalablement étalonné, est i mplanté autour de la veine porte entre le foie et la jonction de la veine gastroplénique au tronc mésentérique. Chaque animal est en outre muni d'une canule du rumen.

- Mesures. - Lors des mesures, le capteur est relié à un débitmètre Statham (type SP 2202) ; l'obtention du zéro de référence, correspondant à un débit nul, ne nécessite pas l'occlusion mécanique de la veine. Le principe de fonctionnement de cet appareil a été décrit antérieurement (Gordon, 1971). Le débitmètre est réglé de façon à éliminer au maximum les signaux parasites et le débit est mesuré avec une constante de temps de $5 \mathrm{sec}$. Quinze mesures en continu sont effectuées sur 4 chèvres durant environ $7 \mathrm{~h}$ après le repas du matin. $100 \mathrm{~g}$ de foin haché sont distribués à l'auge, puis 15 min plus tard $400 \mathrm{~g}$ d'un aliment expérimental granulé et grossièrement broyé sont introduits dans le rumen par la canule. Deux régimes expérimentaux comportant 4 p. 100 d'urée et différant par la nature de leur fraction a mylacée sont utilisés. Chaque animal est accoutumé au régime qui lui a été attribué pendant 2 semaines après l'opération. Les valeurs de débit relevées sur l'enregistrement sont corrigées en fonction de l'étalonnage de la sonde ef de l'hématocrite des animaux.

\section{Résultats.}

1. - Evolution de la mesure du débit après l'opération. - Immédiatement après l'opération, le débit sanguin est difficile à mesurer en raison d'un contact insuffisant entre le capteur et la veine. Les valeurs obtenues sont en général inférieures à 
- D'un jour à l'autre la valeur moyenne du débit sanguin porte peut varier pour un même animal (jusqu'à \pm 25 p. 100) ef les valeurs, même rapportées au poids vif peuvent différer d'un animal à l'autre.

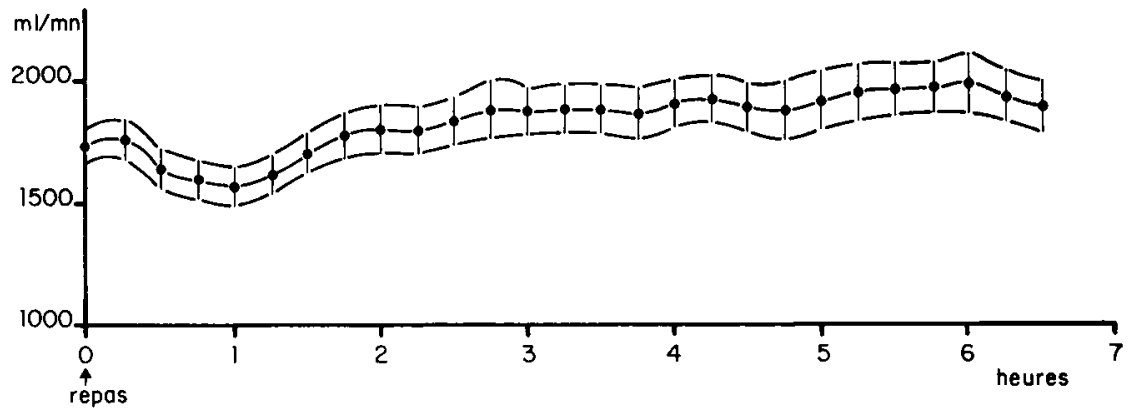

FIG. 2. - Evolution postprandiale du débit sanguin de la veine porte ( \pm erreur standard).

\section{Discussion.}

La valeur moyenne du débit obtenue dans cette expérience est en bon accord avec le chiffre moyen de $39,4 \mathrm{ml} / \mathrm{kg} / \mathrm{min}$ estimé chez le ruminant à partir de la bibliographie (Schambye, 1955 ; Roe, Bergman et Kon, 1966 ; Carr et Jacobson, 1968 ; Katz ef Bergman, 1969 ; Hume, 1971 ; Wangness et McGilliard, 1972 ; Webster et White, 1973 ; Kedenburg et Hubner, 1973 ; Weekes et Webster, 1975 ; Prewitt et al. 1975 ; Sniffen et Jacobson, 1975).

Divers auteurs ont signalé le caractère variable du débit. L'influence de l'anesthésie et du jeûne a été rapportée à plusieurs reprises (Kałz et Bergman 1969 ; Wangness et McGilliard, 1972). Wangness ef McGilliard (1972) n'ont pas remarqué d'évolution du débit dans la journée, mais la plupart des auteurs signalent une augmentation du débit sanguin porte après le repas chez le ruminant (Bensadoun et Reid, 1962 ; Carr ef Jacobson, 1968 ; Katz et Bergman, 1969) et chez le porc (Rérat, 1971). Dans notre expérience, l'influence la plus nette du repas est la baisse du débit pendant $1 \mathrm{~h}$. L'absorption d'ammoniac à la suite de l'hydrolyse dans le rumen de l'urée alimentaire pourrait expliquer ce phénomène : ce métabolite apparaît rapidement dans le sang porte et peut provoquer une somnolence chez les animaux (Lewis, Hill et Annison, 1957) ainsi que nous l'avons constaté dans beaucoup de nos essais. Cependant, cerfaines variations du débit du sang porte du porc (Rérat et al., 1976), chez lequel l'ammoniac ne peut être mis en cause, présentent une grande analogie avec celles que nous avons obtenues. Ce point sera précisé ultérieurement.

En raison des méthodes de mesure de débit généralement utilisées, peu d'auteurs ont signalé des variations rapides de débit : celles-ci auraient été mises en évidence par Schenk ef al. (1960) chez l'homme et Hopkinson et Schenk (1968) obtiennent chez le chien des enregistrements assez semblables aux nôtres. Ces variations rapides du débit peuvent expliquer certaines difficultés rencontrées par les utilisateurs de méthodes de mesure de débit par injection d'un traceur dans la veine porte. 
Les variations de débit que nous avons observées pour un même animal d'un jour à l'autre et entre les animaux confirment les travaux antérieurs (Katz et Bergman, 1969 ; McGilliard, Thorp and Thorp, 1971 ; Wangness et McGilliard, 1972).

\section{Conclusion.}

Le caractère très variable du débit sanguin porte rend nécessaire sa mesure en continu lors de l'estimation quantitative du passage des nutriments dans le sang porte à partir du tube digestif. L'emploi d'un débitmètre électromagnétique permet d'obtenir, sans perturber les animaux, des mesures satisfaisantes chez la chèvre non anesthésiée sur des périodes n'excédant pas 5 semaines.

Commission CNERNA Digestion-Absorption/Association des Physiologistes, Paris 5-6 octobre 1978.

\section{Références}

BENSADOUN A., REID J. T., 1962. Estimation of rate of portal blood flow in ruminants : effect of feeding, fasting and anesthesia. J. Dairy Sci, 45, 540-543.

CARR S. B., JACOBSON D. R., 1968. Method for measurement of gastrointestinal absorption in normal animals combining portal-carotid differences and telematerej, portal flow by doppler-shift. J. Dairy Sci., 51, 721-729.

GORDON A. S., 1971. Practical aspects of blood flow measurement. Statham Instruments Inc. Oxnard USA.

HOPKINSON B. R., SCHENK W. G., 1968. The electromagnetic measurement of liver blost flow and cardiac output on conscious dogs during feeding and exercice. Surgery, 63, 970-975.

HUME J. D., 1971. Amino acid absorption in sheep fed alfafa. J. Anim. Sci., 33, 287 (abstr.).

KATZ M. L., BERGMAN E. N., 1969. Simultaneous measurements of hepatic and portal veinous blood flow in the sheep and dog. Am. J. Physiol., 216, 946-952.

KEDENBURG V. C. P., HÜBNER H., 1973. Stickstoffresorption bai Schafen nach Hardstolf Fülterung. 1. - Mitteilung. Z. Tierphysiol. Tierernähr. Futfermiffelkde, 32, 6 4-73.

LEWIS D., HILL K. J., ANNISON E. F., 1957. Studies on the portal blood of sheep. I. Absorplion of ammonia from the rumen of the sheep. Bioch. J. 66, 587-592.

MCGILLIARD A. D., THORP J. W., THORP S. L., 1971. Variation on portal bloof flow measuref by dye-dilution in young calves. J. Dairy Sci., 54, 247-251.

PREWITT L. R., JACOBSON D. R., HEMKEN R. W., SCHELLING G. D., HATTON R. H., 1975. Amino acid absorption by portal jugular venous differences in sheep fed two maturities of alfafa hay. J. Anim. Sci., 41, 1722-1727.

RÉRAT A., 1971. Mesure du débit de sang dans la veine porte à l'aide d'un débitmètre électromagnétique chez le porc. Ann. Biol, anim. Bioch. Biophys., 11, 175-180.

RÉRAT A., CORRING T., LAPLACE J. P., 1976. Protein digestion and absorption, 97-138. In COLE D. J. A., BOORMAN K. N., BUTTERY P. J., LEWIS D., NEALE R. J., SWAN H., Protein mefabolism and nutrition. Eur. Ass. Anim. Prod., Publ. n० 6, Butterworths, London.

ROE W. E., BERGMAN E. N., KON K., 1966. Absorption of ketone bodies and other metabolites via the portal blood of sheep. Am. J. vet. Res., 27, 729-736.

SCHAMBYE P. 1955. Experimental estimation of the portal vein blood flow in sheep. I. Chronic experiments in cannulated sheep applying infusion and injection methods. Nord. vef. Med., 7, 961-975.

SCHENK W. G., MENNO A. D., ANDERSEN M. N., MURRAY N. A., DRAPANAS T., 1960. Application of the electromagnetic flowmeter to vascular studies in human patients. Surgery, 48, 211-220. 
SNIFFEN C. J., JACOBSON D. R., 1975. Net amino acid absorption in steers fed alfafa hay cut at two stages of maturity. J. Dairy Sci, 58, 371-383.

WANGNESS P. J., McGILLIARD A. D., 1972. Measurement of portal blood flow in calves by dye dilution. J. Dairy Sci., 55, 1439-1446.

WEBSTER A. J. F., WHITE F., 1973. Portal blood flow and heat production in the digestive tract of sheep. Br. J. Nutr., 29, 279-293.

WEEKES T. E. C., WEBSTER A. J. F., 1975. Metabolism of propionate in the tissues of the sheep gut. Br. J. Nutr., 33, 425-437. 